\title{
X-ray spectroscopy of galaxy clusters: beyond the CIE modeling
}

\author{
Liyi Gu · Irina Zhuravleva · Eugene \\ Churazov . Frits Paerels . Jelle \\ Kaastra · Hiroya Yamaguchi
}

Received: date / Accepted: date

\begin{abstract}
X-ray spectra of galaxy clusters are dominated by the thermal emission from the hot intracluster medium. In some cases, besides the thermal component, spectral models require additional components associated, e.g., with

L. $\mathrm{Gu}$

RIKEN High Energy Astrophysics Laboratory, 2-1 Hirosawa, Wako, Saitama 351-0198, Japan

SRON Netherlands Institute for Space Research, Sorbonnelaan 2, 3584 CA Utrecht, the Netherlands

E-mail: liyi.gu@riken.jp

I. Zhuravleva

Kavli Institute for Particle Astrophysics and Cosmology, Stanford University, 452 Lomita Mall, Stanford, California 94305-4085, USA

Department of Physics, Stanford University, 382 Via Pueblo Mall, Stanford, California 94305-4060, USA

E. Churazov

Max-Planck-Institut für Astrophysik, Karl-Schwarzschild-Strasse 1, 85741 Garching, Germany

Space Research Institute (IKI), Profsoyuznaya 84/32, Moscow 117997, Russia

F. Paerels

Columbia Astrophysics Laboratory and Department of Astronomy, Columbia University, 538 W. 120th St., New York, NY 10027, USA

J. Kaastra

SRON Netherlands Institute for Space Research, Sorbonnelaan 2, 3584 CA Utrecht, the Netherlands

Leiden Observatory, Leiden University, Niels Bohrweg 2, 2300 RA Leiden, the Netherlands

H. Yamaguchi

Institute of Space and Astronautical Science (ISAS), Japan Aerospace Exploration Agency (JAXA), Kanagawa 252-5210, Japan
\end{abstract}


resonant scattering and charge exchange. The latter produces mostly underluminous fine spectral features. Detection of the extra components therefore requires high spectral resolution. The upcoming X-ray missions will provide such high resolution, and will allow spectroscopic diagnostics of clusters beyond the current simple thermal modeling. A representative science case is resonant scattering, which produces spectral distortions of the emission lines from the dominant thermal component. Accounting for the resonant scattering is essential for accurate abundance and gas motion measurements of the ICM. The high resolution spectroscopy might also reveal/corroborate a number of new spectral components, including the excitation by non-thermal electrons, the deviation from ionization equilibrium, and charge exchange from surface of cold gas clouds in clusters. Apart from detecting new features, future high resolution spectroscopy will also enable a much better measurement of the thermal component. Accurate atomic database and appropriate modeling of the thermal spectrum are therefore needed for interpreting the data.

Keywords Galaxies: clusters: general - Galaxies: clusters: intracluster medium - Techniques: spectroscopic $\cdot$ X-rays: galaxies: clusters

\section{Introduction}

Galaxy clusters are the largest bound systems in the Universe. The dominant baryonic matter in clusters is in the form of the intracluster medium (hereafter ICM), a tenuous $\left(10^{-5}-10^{-2} \mathrm{~cm}^{-3}\right)$, hot $\left(10^{7-8}\right.$ Kelvin), and large scale $(\sim \mathrm{Mpc})$ plasma in quasi-hydrostatic equilibrium within the dark matter potential. ICM reveals itself by emitting mostly thermal X-rays, which has been extensively studied for assessing the physical properties of the ICM, and for tracing the cosmological structure formation in the Universe.

Many achievements in the studies of ICM were made possible by the advent of X-ray spectroscopic instruments. The line emission of highly-ionized iron was first discovered with the Ariel $\mathrm{V}$ spectrum (spectral resolution $R \sim 6$ ) of the Perseus cluster, establishing the thermal origin of the ICM (Mitchell et al., 1976). The improved spectral resolution with CCD-type spectrometers $(R=10-60)$ led to the discovery of other fundamental elements (O, $\mathrm{Ne}, \mathrm{Mg}$, $\mathrm{Si}, \mathrm{S}, \mathrm{Ar}, \mathrm{Ca}$, and Ni) in the ICM (Mushotzky et al., 1996). The reflection grating spectrometer $(R=50-100$ for spatially extended sources) onboard $X M M-N e w t o n$ made a breakthrough in the soft X-ray spectroscopy. One of its major discoveries is the lack of massive accumulation of the cold gas in cool core clusters (Peterson et al., 2001). More recently, X-ray micro-calorimeters have been developed. The micro-calorimeter onboard Hitomi $(R \sim 1250)$ measured directly velocities of ICM random motions and detected the Fe-peak elements $\mathrm{Cr}, \mathrm{Mn}$, and Ni for the first time (Hitomi Collaboration et al. 2016, 2017).

ICM is far from being quiescent. High-resolution imaging of cluster cores revealed the presence of optical-line emitting filaments, cavities, and weak shocks possibly created by the AGN feedback; on larger scales, radio-luminous shocks by mergers have been observed. The implied energetic thermodynamical 
processes are expected to strongly affect the cluster evolution. Some of the non-thermal processes could, in theory, be detected by X-ray spectrometers, as they would produce characteristic features in the spectrum. In practice, however, firm detections with present X-ray instruments are still limited, as the X-ray signatures are often faint and tend to be swamped by the dominant ICM thermal signal.

Future high-resolution X-ray spectrometers, e.g., the X-ray imaging and spectroscopy mission (XRISM, launch 2021) and Athena (launch 2030), will explore the ICM physics beyond the current standard thermal view. In this review we summarize effects that introduce additional features in the spectra of galaxy clusters and can be observed with future X-ray spectrometers. In particular, the resonant scattering effect (\$2) will be possible to detect in many clusters, and will be widely used as a proxy of random motion in the ICM. In addition, the presence of non-thermal ( $\$ 3$, non-equilibrium ionization (\$4), and charge exchange $(\$ 5$ phenomena could be addressed through measurements of particular line emission.

Apart from detecting new X-ray features, it is also important to model properly the known thermal emission. As more high-resolution X-ray spectra become available, more accurate atomic data and plasma models will be urgently needed. Here, we also review the status of current atomic codes (\$6), the achievements and challenges in the work with the Hitomi spectrum of the Perseus cluster.

\section{Resonance scattering}

Resonant scattering (RS) is a process of absorption and re-emission of a photon, having the energy close to a resonant transition of an atom or ion. The resonant scattering is especially interesting in the context of galaxy clusters because it can affect measured element abundances in the cluster cores and, also, serve as an independent proxy for gas motions (Gilfanov, Sunyaev \& Churazov, 1987, Churazov et al., 2010). Measuring line width using high resolution $\mathrm{X}$-ray spectra is the most direct way of probing the motions, but for extended sources such data are still sparse (Hitomi Collaboration et al. 2016). Moreover, given the relatively low energy resolution of future X-ray calorimeters at the Fe-L energies (Takahashi et al. 2016), resonance scattering will remain an important tool for measuring gas motion in elliptical galaxies and galaxy groups, which emit primarily in the soft X-ray band.

\subsection{Optical depth and line width}

Despite their large size $(L \sim \mathrm{Mpc})$, galaxy clusters contain very low density gas $\left(n_{e} \sim n_{p} \sim 10^{-5}-10^{-2} \mathrm{~cm}^{-3}\right)$. This results in a small Thomson optical depth $\left(\tau_{T} \lesssim 10^{-3}\right)$ and essentially zero free-free opacity in X-rays. However, the optical depth near the resonant transition of astrophysically abundant 
elements can be of the order of unity (Gilfanov, Sunyaev \& Churazov, 1987).

The optical depth at the center of a line can be written as

$$
\tau_{R S}=n_{p} L Z \delta_{i}(T) \frac{\sqrt{\pi} h r_{e} c f}{\Delta E_{D}},
$$

where $L$ is the characteristic size, $Z=\frac{n_{Z}}{n_{H}}$ is the abundance of an element with respect to hydrogen; $\delta_{i}(T)$ is the fraction of a given ion, set by the ionization balance; $h, r_{e}, c$ are the Planck constant, classical electron radius, and the speed of light, respectively; $f$ is the absorption oscillator strength of a given atomic transition. $\Delta E_{D}$ is the Doppler width of the line, set by the ion thermal velocities and by the "turbulent" velocities within the observed region

$$
\Delta E_{D}=E_{0}\left[\frac{2 k T_{e}}{A m_{p} c^{2}}+\frac{2 V_{1, t u r b}^{2}}{c^{2}}\right]^{1 / 2},
$$

where $A$ is the atomic weight of the corresponding element, $m_{p}$ is the proton mass and $V_{1, t u r b}$ is the RMS of gas velocities in one direction. A natural width of the line could also be included in the expression for $\Delta E_{D}$, but for the ICM conditions it is usually sub-dominant.

Three things are immediately clear from eqs. (1) and (2). Firstly, it is likely that due to the factor $Z \delta_{i}(T)$ in eq. (1), the optical depth will be the largest for [He-] and [Ne-] like ions of the most abundant elements.

Secondly, in the expression for the line width, the ion thermal broadening is "attenuated" by the atomic weight $A$, which makes $\Delta E_{D}$ and, therefore, the optical depth $\tau_{R S}$, sensitive to the gas motions, even if they are subsonic. Thirdly, the brightest lines in the optically thin plasma are often associated with resonant transitions. Therefore, the very same line photons will be the most strongly affected by the resonant scattering.

\subsection{Phase function and polarization}

For any transition, the resonant scattering can be represented as a combination of two processes: isotropic scattering with a weight $w_{1}$ and Rayleigh scattering with a weight $w_{2}=1-w_{1}$ (Hamilton, 1947). The weights $w_{1}$ and $w_{2}$ depend on the total angular momentum $j$ of the ground level and on the difference between total angular momenta of the excited and ground levels $\Delta j(= \pm 1$ or 0 ). If the initial radiation is unpolarized, the probability of the photon scattering by angle $\theta$ is

$$
P(\mu)=\frac{1}{4 \pi}\left[w_{1}+\frac{3}{4}\left(1+\mu^{2}\right) w_{2}\right],
$$

where $\mu=\cos \theta$. The isotropic component of the phase function does not induce any polarization, while the Rayleigh component does. If polarization is of interest, then a full scattering matrix for Stokes parameters has to be used 


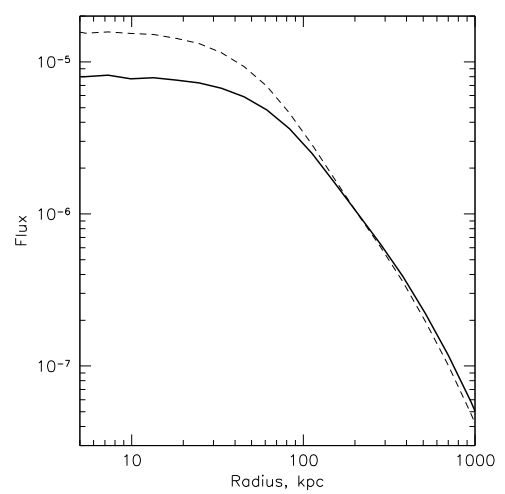

Fig. 1 Radial profiles of the He-like iron $K_{\alpha}$ line with (thick solid line) and without (dashed line) resonant scattering in the Perseus cluster. Resonant scattering suppresses the line intensity in the core and redistributes line photons to larger radii. Pure thermal broadening and a flat radial abundance profile were assumed in this calculation. Adapted from Churazov et al. (2004).

(Hamilton, 1947; Chandrasekhar, 1950). Given that more line photons are generated in the dense cluster cores, the polarization arises ${ }^{1}$ in the cluster periphery, with the polarization plane orthogonal to the radial direction (Sazonov, Churazov \& Sunyaev, 2002). The resonant line of the He-like triplet $w_{1}=0$ and $w_{2}=1$, and, therefore, is the most promising target for future polarimeters. The magnitude of the polarization is sensitive to the line broadening via the optical depth, and, therefore, to the turbulent velocities (Zhuravleva et al. 2010). In many practical applications, one can ignore polarization effects and use an isotropic phase function for all transitions. The errors introduced by this approximation are often smaller than the uncertainties in the model, e.g., an assumption of a spherically symmetric cluster.

\subsection{Surface brightness and line profile}

Apart from the polarization, the resonant scattering leads to (i) a modification of the surface brightness profile of a cluster in the resonant line and (ii) changes in the line spectrum.

The largest optical depth is associated with the densest central part of the cluster. The photons born in this central part are scattered from the line of sight. As a result, the line flux towards the cluster center diminishes, producing a flattened (see Fig 1) surface brightness distribution Gilfanov, Sunyaev \& Churazov, 1987). Due to the conservative nature of resonant scattering, the photons removed from the line of sight towards the cluster center are redistributed to larger projected distances. This effect causes a slight increase

1 for transitions with non-zero Rayleigh component 


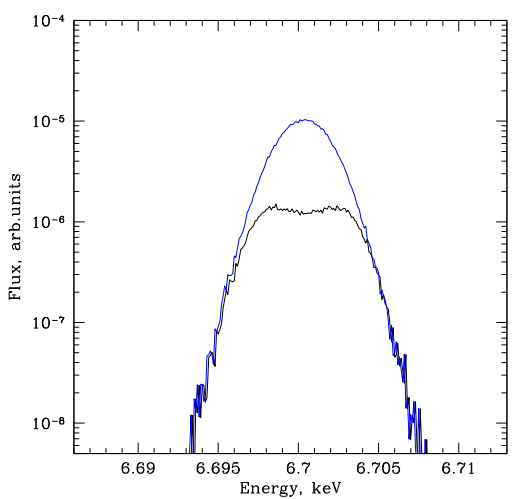

Fig. 2 Simulated spectra of the $6.7 \mathrm{keV}$ line emerging from the core of a cluster. Without scattering the line has a Gaussian shape (top blue curve). Due to the large optical depth in the center of the line, the photons are scattered away from the line of sight, suppressing the line emission close to the center. Adapted from Churazov et al. (2010).

of the surface brightness at larger radii, although this excess emission is more difficult to detect.

Since the radial surface brightness of the line can be extracted from the data with moderate spectral resolution, most of the studies use this approach. In the context of clusters and massive elliptical galaxies the resonant scattering has been most often considered in relation with the "abundance hole" or "abundance anomalies" in the central regions of clusters (e.g. Molendi et al. 1998; Akimoto et al., 1999; Buote, Canizares, \& Fabian, 1999; Böhringer et al. 2001; Dupke \& Arnaud, 2001; Mathews, Buote, \& Brighenti, 2001; Ezawa et al., 2001; Xu et al., 2002, Gastaldello \& Molendi, 2004; Sanders \& Fabian. 2006; Panagoulia, Sanders, \& Fabian, 2015).

Another group of studies was more focused on constraining the turbulent velocities using the line ratios as a proxy to the turbulent broadening of the resonant line (e.g. Churazov et al. 2004; Werner et al., 2009, Zhuravleva et al., 2011; de Plaa et al., 2012, Shang \& Oh, 2013; Pinto et al., 2016; Ogorzalek et al., 2017).

Without the resonant scattering the emitted line spectrum and the optical depth of the line are closely related:

$$
F(E) \propto \tau(E)=\tau_{R S} \times e^{-\frac{\left(E-E_{0}\right)^{2}}{\Delta E_{D}^{2}}} .
$$

Note that this expression ignores Lorentzian wings of the lines and assumes that the line-of-sight gas velocity distribution can be approximated by a Gaussian. Given that the optical depth peaks at the center of the line and goes down quickly in the wings, only the core of the line suffers from the resonant scattering, while the photons in the wings freely escape without any scattering. As a result, the line profile is modified, forming a depression in the center (Gilfanov, Sunyaev \& Churazov, 1987), as shown in Fig 2, For the first time, such 
Table 1 Top: the list of lines in the $5-10 \mathrm{keV}$ energy band in the Perseus cluster with the largest optical depths. Bottom: same for the elliptical galaxy NGC 4636 in the $0.5-1 \mathrm{keV}$ band. Only lines with the optical depth $>0.1$ are shown. The optical depth is calculated assuming zero Mach number and isotropic turbulence with the Mach number 0.2 (0.25) in Perseus (NGC 4636). The Mach number is defined as $M=V_{1, t u r b} / c_{s}$, where $c_{s}$ is the sound speed.

\begin{tabular}{|c|c|c|c|c|c|}
\hline \multicolumn{6}{|c|}{ Perseus Cluster } \\
\hline Ion & $E, \mathrm{keV}$ & Transition & $f$ & $\tau_{\mathrm{M}=0}$ & $\tau_{\mathrm{M}=0.2}$ \\
\hline$\overline{\text { Fe XXIV }}$ & 6.66188 & $1 \mathrm{~s}^{2} 2 \mathrm{~s}\left({ }^{2} \mathrm{~S}_{1 / 2}\right)-1 \mathrm{~s} 2 \mathrm{~s} 2 \mathrm{p}\left({ }^{2} \mathrm{P}_{3 / 2}\right)$ & 0.4892 & 0.216 & 0.080 \\
\hline Fe XXV & 6.66755 & $1 \mathrm{~s}^{2}\left({ }^{1} \mathrm{~S}_{0}\right)-1 \mathrm{~s} 2 \mathrm{p}\left({ }^{3} \mathrm{P}_{1}\right)$ & 0.0579 & 0.170 & 0.063 \\
\hline Fe XXV & 6.70041 & $1 \mathrm{~s}^{2}\left({ }^{1} \mathrm{~S}_{0}\right)-1 \mathrm{~s} 2 \mathrm{p}\left({ }^{1} \mathrm{P}_{1}\right)$ & 0.7192 & 2.100 & 0.780 \\
\hline Fe XXVI & 6.97307 & $1 \mathrm{~s}\left({ }^{2} \mathrm{~S}_{1 / 2}\right)-2 \mathrm{p}\left({ }^{2} \mathrm{P}_{3 / 2}\right)$ & 0.2731 & 0.147 & 0.054 \\
\hline Fe XXV & 7.88153 & $1 \mathrm{~s}^{2}\left({ }^{1} \mathrm{~S}_{0}\right)-1 \mathrm{~s} 3 \mathrm{p}\left({ }^{1} \mathrm{P}_{1}\right)$ & 0.1369 & 0.340 & 0.126 \\
\hline Fe XXV & 8.29548 & $1 \mathrm{~s}^{2}\left({ }^{1} \mathrm{~S}_{0}\right)-1 \mathrm{~s} 4 \mathrm{p}\left({ }^{1} \mathrm{P}_{1}\right)$ & 0.0478 & 0.113 & 0.042 \\
\hline \multicolumn{6}{|c|}{$\overline{\overline{\text { NGC } 4636}}$} \\
\hline$\overline{\text { Ion }}$ & $E, \mathrm{keV}$ & Transition & $f$ & $\tau_{\mathrm{M}=0}$ & $\tau_{\mathrm{M}=0.25}$ \\
\hline O VIII & 0.65349 & $1 \mathrm{~s}\left({ }^{2} \mathrm{~S}_{1 / 2}\right)-2 \mathrm{p}\left({ }^{2} \mathrm{P}_{1 / 2}\right)$ & 0.1385 & 0.409 & 0.210 \\
\hline O VIII & 0.65368 & $1 \mathrm{~s}\left({ }^{2} \mathrm{~S}_{1 / 2}\right)-2 \mathrm{p}\left({ }^{2} \mathrm{P}_{3 / 2}\right)$ & 0.2771 & 0.819 & 0.420 \\
\hline Ne IX & 0.92200 & $1 \mathrm{~s}^{2}\left({ }^{1} \mathrm{~S}_{0}\right)-1 \mathrm{~s} 2 \mathrm{p}\left({ }^{1} \mathrm{P}_{1}\right)$ & 0.7417 & 0.348 & 0.163 \\
\hline Fe XVII & 0.72714 & $2 \mathrm{~s}^{2} 2 \mathrm{p}^{6}\left({ }^{1} \mathrm{~S}_{0}\right)-2 \mathrm{~s}^{2} 2 \mathrm{p}^{5} 3 \mathrm{~s}(3 / 2,1 / 2)_{1}$ & 0.1257 & 0.340 & 0.104 \\
\hline Fe XVII & 0.81243 & $2 \mathrm{~s}^{2} 2 \mathrm{p}^{6}\left({ }^{1} \mathrm{~S}_{0}\right)-2 \mathrm{~s}^{2} 2 \mathrm{p}^{5} 3 \mathrm{~d}\left({ }^{3} \mathrm{D}_{1}\right)$ & 0.6391 & 1.545 & 0.471 \\
\hline Fe XVII & 0.82579 & $2 \mathrm{~s}^{2} 2 \mathrm{p}^{6}\left({ }^{1} \mathrm{~S}_{0}\right)-2 \mathrm{~s}^{2} 2 \mathrm{p}^{5} 3 \mathrm{~d}\left({ }^{1} \mathrm{P}_{1}\right)$ & 2.4938 & 5.933 & 1.808 \\
\hline Fe XVII & 0.89681 & $2 s^{2} 2 p^{6}\left({ }^{1} S_{0}\right)-2 s 2 p^{6} 3 p\left({ }^{1} P_{1}\right)$ & 0.2869 & 0.628 & 0.192 \\
\hline Fe XVIII & 0.85306 & $2 \mathrm{~s}^{2} 2 \mathrm{p}^{5}\left({ }^{2} \mathrm{P}_{3 / 2}\right)-2 \mathrm{~s}^{2} 2 \mathrm{p}^{4} 3 \mathrm{~d}\left({ }^{2} \mathrm{~F}_{5 / 2}\right)$ & 0.2045 & 0.417 & 0.127 \\
\hline Fe XVIII & 0.86262 & $2 \mathrm{~s}^{2} 2 \mathrm{p}^{5}\left({ }^{2} \mathrm{P}_{3 / 2}\right)-2 \mathrm{~s}^{2} 2 \mathrm{p}^{4} 3 \mathrm{~d}\left({ }^{2} \mathrm{D}_{5 / 2}\right)$ & 0.3083 & 0.622 & 0.190 \\
\hline Fe XVIII & 0.86970 & $2 \mathrm{~s}^{2} 2 \mathrm{p}^{5}\left({ }^{2} \mathrm{P}_{3 / 2}\right)-2 \mathrm{~s}^{2} 2 \mathrm{p}^{4} 3 \mathrm{~d}\left({ }^{3} \mathrm{~S}_{1 / 2}\right)$ & 0.2276 & 0.456 & 0.139 \\
\hline Fe XVIII & 0.87264 & $2 \mathrm{~s}^{2} 2 \mathrm{p}^{5}\left({ }^{2} \mathrm{P}_{3 / 2}\right)-2 \mathrm{~s}^{2} 2 \mathrm{p}^{4} 3 \mathrm{~d}\left({ }^{2} \mathrm{P}_{3 / 2}\right)$ & 0.6116 & 1.219 & 0.372 \\
\hline Fe XVIII & 0.87264 & $2 \mathrm{~s}^{2} 2 \mathrm{p}^{5}\left({ }^{2} \mathrm{P}_{3 / 2}\right)-2 \mathrm{~s}^{2} 2 \mathrm{p}^{4} 3 \mathrm{~d}\left({ }^{2} \mathrm{D}_{5 / 2}\right)$ & 0.9295 & 1.854 & 0.565 \\
\hline Fe XIX & 0.91718 & $2 s^{2} 2 p^{4}\left({ }^{3} P_{2}\right)-2 s^{2} 2 p^{3} 3 d(5 / 2,5 / 2)_{3}$ & 0.7438 & 0.854 & 0.260 \\
\hline Fe XIX & 0.91861 & $2 \mathrm{~s}^{2} 2 \mathrm{p}^{4}\left({ }^{3} \mathrm{P}_{2}\right)-2 \mathrm{~s}^{2} 2 \mathrm{p}^{3} 3 \mathrm{~d}(5 / 2,3 / 2)_{3}$ & 0.3781 & 0.434 & 0.132 \\
\hline Ni XIX & 0.99706 & $2 \mathrm{~s}^{2} 2 \mathrm{p}^{6}\left({ }^{1} \mathrm{~s}_{0}\right)-2 \mathrm{~s}^{2} 2 \mathrm{p}^{5} 3 \mathrm{~d}(1 / 2,3 / 2) 1$ & 2.5214 & 0.324 & 0.097 \\
\hline
\end{tabular}

modification in a galaxy cluster has been found in the Hitomi data (Hitomi Collaboration et al., 2018, see $\$ 2.5$ below).

\subsection{Modeling resonant scattering}

Spectral and spatial distortions due to the resonant scattering have been estimated analytically in a small- $\tau$ limit for isothermal gas (Gilfanov, Sunyaev \& Churazov, 1987, Sazonov, Churazov \& Sunyaev, 2002). For real objects, it is necessary to perform radiative transfer simulations, which take into account gas temperature gradient and multiple scatterings of photons. The typical optical depth in individual lines is $\lesssim$ few in galaxy clusters, groups and elliptical galaxies (see Table 1). Therefore, radiative transfer effects can be efficiently modeled following a Monte Carlo approach. In simulations, the act of scattering can be modeled in full details. First, a photon with the energy $E$ is absorbed by an ion, moving with such velocity that in the frame of the ion, the photon energy is equal to the energy $E_{0}$ of the line, i.e.,

$$
E=E_{0}\left[1+\frac{\left(\mathbf{V}_{\text {ion }} \mathbf{m}\right)}{c}\right]
$$


where $\mathbf{V}_{\text {ion }}$ and $\mathbf{m}$ are the ion velocity and the direction of the photon propagation in the cluster frame, respectively ${ }^{2}$. Given that the above expression depends only on the scalar product $\left(\mathbf{V}_{\text {ion }} \mathbf{m}\right)$, there are no constraints on the two components of $\mathbf{V}_{\text {ion }}$ that are perpendicular to $\mathbf{m}$. These two components are drawn from a Gaussian distribution with the width set by the Doppler line width. The photon is scattered in the frame moving with ion (according to the relevant isotropic and/or dipole phase function) and transformed back to the cluster frame. After each scattering the weight, initially assigned as unity to each photon, is reduced by a factor of $\left(1-e^{-\tau}\right)$, where $\tau$ is the optical depth in the direction of photon propagation. The scattering process repeats until the weight drops below the set minimal value.

Two independent approaches of simulating resonant scattering have been implemented: using a Geant $3^{3}$ toolkit for the simulations of the passage of particles through matter (see e.g. Hitomi Collaboration et al., 2018) and Monte Carlo simulations tailored for X-ray photons propagating through gas-rich atmospheres (e.g. Sazonov, Churazov \& Sunyaev, 2002; Hitomi Collaboration et al., 2018, and references therein). The latter simulations considered, initially, only individual, optically-thick lines, assumed a spherically-symmetric geometry and an isotropic turbulence (Sazonov, Churazov \& Sunyaev, 2002, Churazov et al., 2004). These simulations have been later extended to study the resonant scattering in galaxy clusters taken from cosmological simulations (Zhuravleva et al., 2010), in anisotropic velocity field (Zhuravleva et al., 2011) and in a broad range of energies, producing spectral models suitable for direct comparison with observed spectra (Zhuravleva et al., 2013).

Taking line emissivities and continuum emission from the latest AtomDB (v3.0.9) model of an optically thin plasma, we updated the results of the resonant scattering simulations in the core of the Perseus cluster (for earlier results see Zhuravleva et al., 2013). Fig. 3 shows projected spectra with and without resonant scattering in the $0.5^{\prime}-2^{\prime}$ annulus, the ratio of these spectra and the optical depth as a function of photon energy. The spectra are shown for the He-like Fe line complex. Middle and bottom panels show the results when gas is static ( $M=0$, navyblue curves) and when turbulence is present in the gas with the Mach number of one-component velocity $M=V_{1, \text { turb }} / c_{s}=0.2$ (dodgerblue). Several lines in the He-like Fe triplet are affected by scattering. The resonant Fe line (W) at $6.7 \mathrm{keV}$ has the largest optical depth $\tau \sim 2$ $(\mathrm{M}=0)$ and $\sim 0.8(\mathrm{M}=0.2)$. As the result of scattering, the flux in this line is suppressed by a factor of $2(1.3)$ when $M=0(M=0.2)$ and the wings of the line become stronger (little troughs in the middle panel in Fig. 3). Modeling such distorted line with a Gaussian will effectively give a larger line width compared to the other lines of the same ion. Intercombination lines $(\mathrm{Y})$ in the Fe triplet are slightly affected by the resonant scattering as well. However, line distortions disappear even for a relatively low turbulence.

\footnotetext{
2 here we assume that $V_{\text {ion }} \ll c$

3 http://geant4.cern.ch
} 


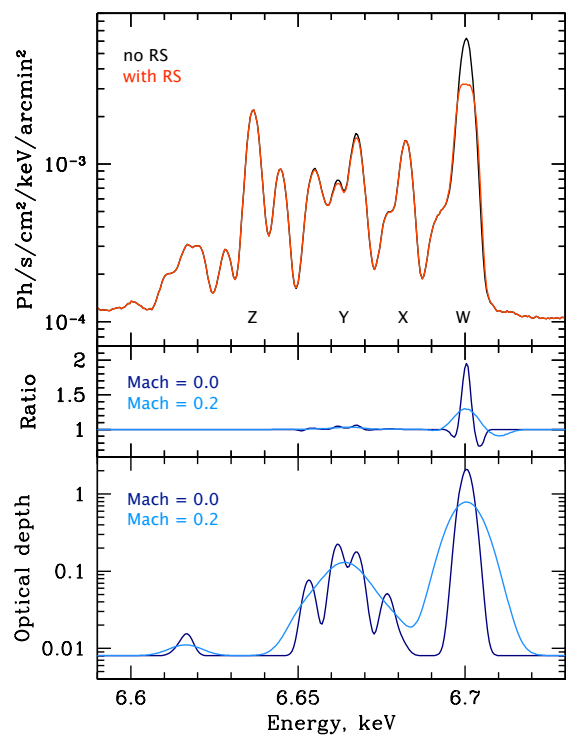

Fig. 3 Resonant scattering in the 0.5' - 2' annulus in the Perseus cluster. Only the spectrum of He-like Fe triplet is shown. Top: spectrum with (red) and without (black) resonant scattering. Middle: ratio of the two models when gas is static $(M=0.0$, navyblue) and turbulence is present in the gas with a Mach number 0.2 (dodgerblue). Bottom: optical depth as a function of photon energy for the two cases of Mach numbers. Adapted from Zhuravleva et al. (2013), updated for the latest AtomDB model (v3.0.9).

Fig. 4 shows the resonant scattering effect in a massive elliptical galaxy NGC 4636. The gas in NGC 4636 is significantly cooler than in Perseus, the average temperature is $<1 \mathrm{keV}$. For such temperatures, the strongest emission lines are the lines of the Ne-like Fe at $15 \AA$ and $17 \AA$. The optical depth of the strongest resonant line at $0.826 \mathrm{keV}$ is $\sim 6$ (Table 1), almost three times larger than the optical depth in the $\mathrm{W}$ line in Perseus. Resonant scattering suppresses the flux in this line by a factor of more than 6 and 2 for the Mach numbers 0 and 0.25 , respectively (Fig. 4). Several other lines strongly affected by the scattering, including the Fe XVIII line at $0.873 \mathrm{keV}$ with the flux suppression by a factor of $\sim 4$ when $M=0$ and the Fe XVII line at $0.812 \mathrm{keV}$ with the suppression by a factor of $\sim 2$ (Fig. 4).

\subsection{Observing resonant scattering}

Spectral distortions due to the resonant scattering effect have been searched in many objects. Using high-resolution RGS/XMM-Newton spectra, a clear evidence of the effect in the Fe XVII line at $15.01 \AA$ has been found in the bright and compact cores of several elliptical galaxies, including NGC 4636, NGC 1404, NGC 5813 and NGC 4472 (Xu et al., 2002; Kahn et al., 2003 Werner et al., 2009, de Plaa et al., 2012). Ahoranta et al. (2016) studied spa- 

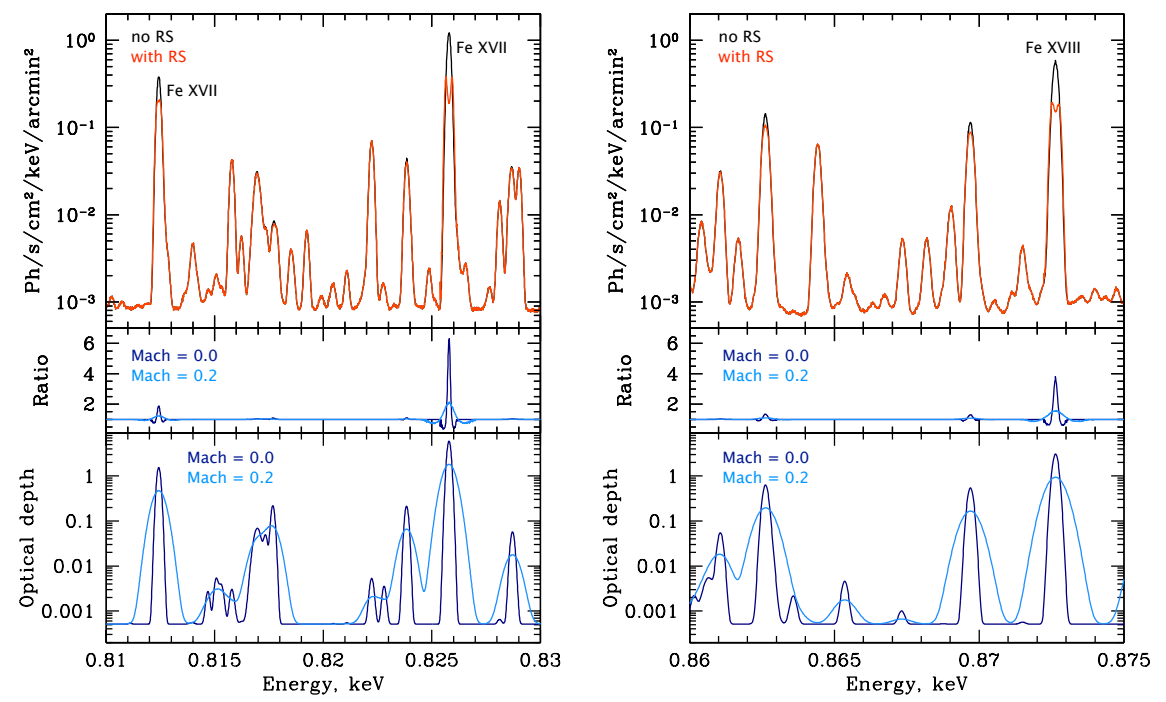

Fig. 4 Resonant scattering in the inner kpc region in NGC 4636. Only the portions of spectra containing lines with the largest optical depth are shown. Color coding is the same as in Fig. 3

tial variations of the Fe XVII optically thin to thick line ratio in NGC 4636, suggesting that the resonant scattering effect is smaller in the southern and stronger in the northern regions of the galaxy. Recently, Ogorzalek et al. (2017) performed a detailed analysis of the resonant scattering detected with the RGS observations in a sample of 22 nearby, giant elliptical galaxies and, combining the analysis with the Doppler broadening technique, constrained the turbulent velocity in 13 objects. There are observational indication of the resonant scattering in a cooler gas component with the temperature $\sim 2 \cdot 10^{6} \mathrm{~K}$, which is traced by the OVII emission lines. In several elliptical galaxies, the observed OVII resonant-to-forbidden line ratio is decreased, which could be due to the resonant scattering or charge exchange (Pinto et al. 2016$)$. The same authors found that OVII is mainly detected in galaxies, where the Fe XVII resonant line is suppressed by the resonant scattering.

Despite many attempts to detect the resonant scattering effect in the Perseus cluster (e.g. Molendi et al., 1998; Ezawa et al., 2001; Churazov et al. 2004) and other clusters (Kaastra et al., 1999:|Akimoto et al. |2000| Mathews, Buote, \& Brighenti, 2001:|Sakelliou et al., 2002; Sanders \& Fabian, 2006), the conclusions remained controversial. The energy resolution of the CCD-type spectrometers is not sufficient to distinguish between spectral distortions due to the resonant scattering, variations of gas temperature and metallicity and gas motions (Zhuravleva et al., 2013). Also, flux suppression due to the resonant scattering in the resonant Fe XXV line is often weaker than in the resonant Fe XVII line in elliptical galaxies. Recent RGS observations of the Perseus cluster show the Fe XVII forbidden-to-resonant line ratio is signifi- 
cantly higher than predicted from optically thin plasma, suggesting that the resonant scattering might be important in the cluster core (Pinto et al. 2016).

The resonant scattering controversy in the Perseus cores has been recently resolved with the Hitomi observations. Due to the superb energy resolution of $5 \mathrm{eV}$ at $6 \mathrm{keV}$ of the soft X-ray Spectrometer on-board Hitomi, the individual spectral lines have been resolved (Hitomi Collaboration et al., 2018) and the suppression of the resonant Fe XXV line has been measured robustly (Hitomi Collaboration et al. 2018). Fig. 5 shows the He-like Fe triplet observed in the Perseus inner $\sim 20 \mathrm{kpc}$ region and modeled with an optically thin plasma model from AtomDB, accounting for the flux suppression in the resonant $\mathrm{W}$ line. The $\mathrm{W}$ line flux is suppressed by a factor of $\sim 1.3$ in the innermost $\sim$ $40 \mathrm{kpc}$ and by a factor of $\sim 1.15$ in the region $\sim 30-100 \mathrm{kpc}$ away from the cluster center. Both, the amplitude of the flux suppression and the variations of the suppression with the projected distance from the cluster center, are in excellent agreement with the theoretical predictions of the resonant scattering. Also, an additional broadening 4 of the resonant line compared to other lines from the same ion has been observed, supporting a non-Gaussian shape of the W line.

\subsection{Uncertainties in the atomic physics}

The resonant scattering is measured by comparing the observed line ratios with the models, which introduces systematic uncertainties from the underlying atomic physics. The uncertainties mainly originate in two parameters: the oscillator strengths of the resonant lines, and the model intensity ratio of the resonant-to-forbidden lines.

A systematic estimate of the atomic uncertainties was given in Hitomi Collaboration et al. (2018), for the study of the Perseus spectrum $(k \mathrm{~T} \approx 4 \mathrm{keV})$ taken with the Hitomi satellite. The oscillator strength of the Fe XXV He $\alpha$ resonant transition was found to be well determined, introducing a systematic error of $<5 \%$. The resonant-to-forbidden line ratio has a more complex nature. The uncertainty is contributed by three components, the errors in the line formation kinetics, errors associated with the unresolved satellite lines, and errors from the possible charge exchange recombination. The first component can be assessed with the ground laboratory measurement at the electron beam ion trap (EBIT) device. By comparing the current plasma code (AtomDB/APEC and SPEX) with the EBIT line ratio for a $4 \mathrm{keV}$ Maxwellian plasma reported in $\mathrm{Gu}$ et al. (2012), the error associated with the line formation was found to be $\sim 6 \%$. The second component was estimated by comparing the unresolved satellite lines obtained with the AtomDB/APEC, SPEX, and the ground experiments. The total error on the Fe XXV resonant-to-forbidden line ratio is about $4 \%$ for a $4 \mathrm{keV}$ plasma. As for the third component, the recombination from charge exchange between Fe XXVI and neutral atom can suppress the

4 when the line is approximated with a Gaussian 

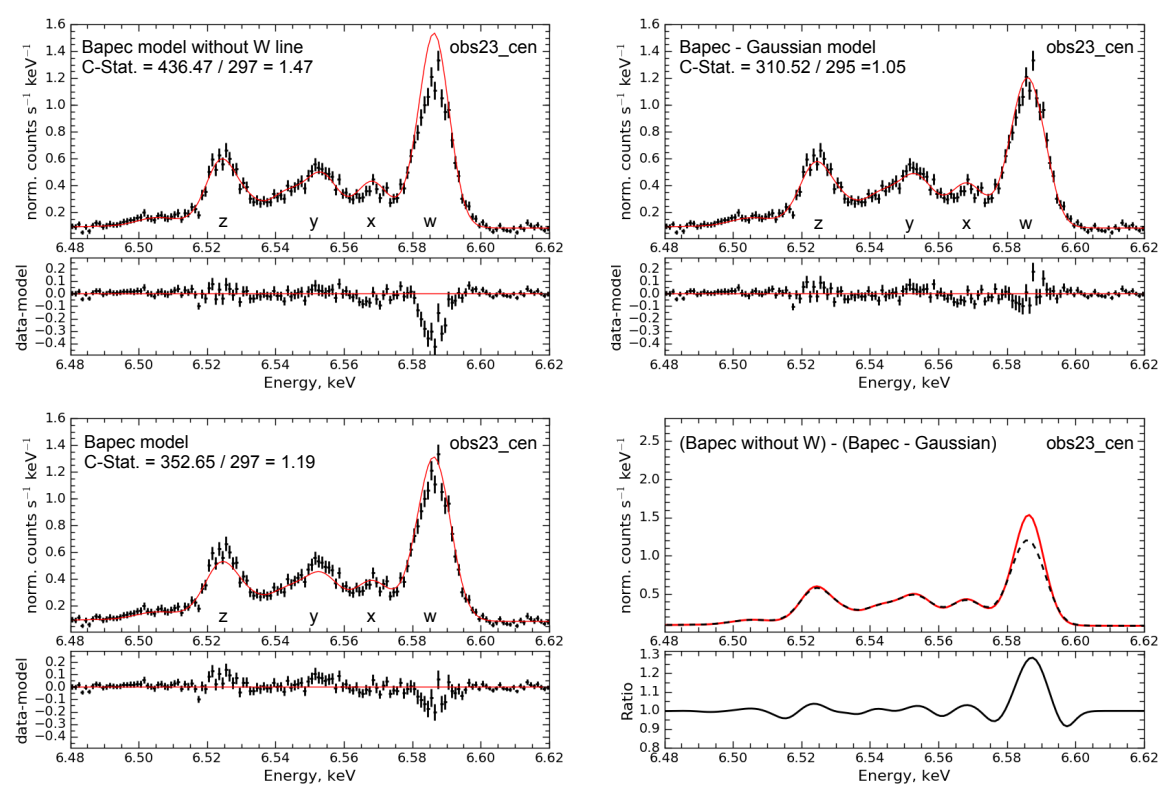

Fig. 5 Observed flux suppression in the strongest line of He-like Fe XXV (w) in the Perseus Cluster observed in inner $\sim 20 \mathrm{kpc}$ region. Black points show the Hitomi data; red lines in the corresponding panels show the best-fitting models. Top left: the spectrum is fitted with the bapec model from AtomDB, excluding the $\mathrm{w}$ line from the data; top right: the same spectrum is fitted with the bapec model and a Gaussian component centered at the energy of the $\mathrm{w}$ line, the normalization of the Gaussian model is allowed to be negative; bottom left: the same spectrum fitted with the bapec model. The comparison of the models from the top left (solid) and right (dashed) panels is shown in the bottom right panel. Adapted from Hitomi Collaboration et al. (2018)

resonant-to-forbidden line ratio by $\sim 6 \%$ in the core of the Perseus cluster. A detailed review on the charge exchange process will be given in $\$ 5$.

Combining the errors associated with the oscillator strength and resonantto-forbidden line ratio, the total systematic uncertainty for the resonant scattering in the Perseus cluster is about $10 \%$. For the cooler systems, such as elliptical galaxies, the atomic errors can be larger than those for the hot clusters. As shown in Ogorzalek et al. (2017), the error from the line formation kinetics on the Fe XVII resonant-to-forbidden line ratio is $\sim 15 \%$. Future theoretical calculations and targeted experiments providing better agreement on the atomic properties are needed to match the precision of the existing and future high spectral resolution X-ray missions.

\section{Non-thermal effects}

Non-thermal electrons can be produced in clusters of galaxies in various ways. Near the interface of cold and hot gas, thermal electrons from the hot gas may penetrate into the cold gas and constitute there a population of super-thermal 


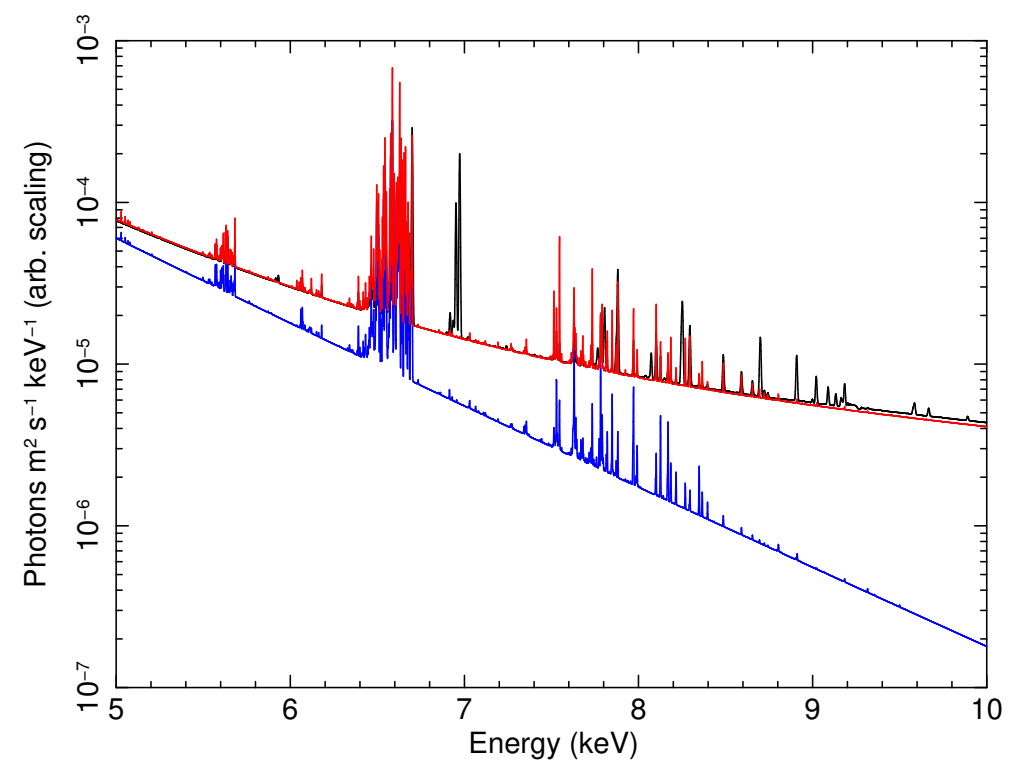

Fig. 6 Blue curve: collisional ionisation equilibrium (CIE) $k T=1 \mathrm{keV}$ spectrum; Black curve: sum of a $k T=1 \mathrm{keV}$ CIE spectrum and a $k T=10 \mathrm{keV}$ CIE spectrum, with the hotter component having $1 \%$ of the emission measure of the colder component; Red curve: spectrum of a plasma with $k T=1 \mathrm{keV}$ thermal electron distribution plus a $1 \%$ contribution from a $k T=10 \mathrm{keV}$ electron distribution.

electrons. This process only works when the magnetic field geometry allows these electrons to pass. Acceleration of electrons is perhaps the most attractive mechanism to produce non-thermal electrons. This can happen near magnetic neutral points during magnetic reconnection by the $v \times B$ electric fields, or likely more frequently as a product of shocks.

Observing these non-thermal electrons offers direct insight into the physical processes that create them and hence in the physics of the cluster gas. One may distinguish three ways in which non-thermal electrons affect the X-ray spectrum of hot gas (Kaastra et al., 2009): 1) the production of a non-thermal bremsstrahlung tail to the continuum spectrum, 2) a shift in the ionisation balance towards higher ionisation, and 3) modified line intensities due to resonant processes.

Non-thermal bremsstrahlung tails can be detected by searching for excess continuum emission at high energies. We refer to Rephaeli et al. (2008) for a review on that process. The effects on the ionisation balance are in general not very large, but should be taken into account, unless the non-thermal electrons are short-lived, in which case they might affect the instantaneous spectrum, but the plasma may not have had enough time to adjust. The SPEX package (Kaastra et al., 1996) allows to treat both cases.

Non-thermal electrons also affect line ratios. This happens for all resonant processes, like dielectronic recombination or collisional excitation resonances. 
Normal excitation processes, like collisional excitation by the hot plasma, have different cross sections as a function of energy, but are possible for all electrons in the plasma. Hence they are usually dominated by the bulk of the (thermal) electrons. Resonances, on the other hand, are only possible for a specific electron energy $E_{r}$. Resonant contributions to the line intensities are thus determined by the relative contribution of the electron distribution at $E_{r}$ versus the bulk of the thermal electrons. For each transition, this ratio is different and thus dielectronic satellite lines offer an excellent opportunity to demonstrate the presence and quantify the amount of non-thermal electrons (Gabriel \& Phillips, 1979).

Technically, the presence of non-thermal electrons in the spectrum is best accounted for by writing the electron distribution as the sum of Maxwellians. This is because the bulk of all atomic processes are characterised by rates that are averaged over a thermal electron distribution. The SPEX package (Kaastra et al., 1996) has implemented the treatment of non-thermal electrons in this way. Recently, Hahn \& Savin (2015) have provided a convenient parameterisation of the so-called kappa-distribution in terms of a sum of Maxwellians.

Care should be taken when interpreting spectra with non-thermal electrons. Fig. 6 illustrates this. The red curve is a spectrum of a $1 \mathrm{keV}$ plasma with an additional non-thermal electron contribution of $1 \%$ with a 10 times higher energy. Compared with a simple $1 \mathrm{keV}$ thermal spectrum (blue curve) the most striking feature is the non-thermal bremsstrahlung tail most visible at the higher energies. In order to demonstrate that this is the spectrum of a plasma with non-thermal electrons, sensitive lines should be used but in addition it should be shown that the spectrum is not simply the sum (or superposition) of two separate plasmas with different temperatures (1 and $10 \mathrm{keV})$ and emission measures (the hotter component $1 \%$ of the colder component); this is the black curve.

It is seen immediately that the main difference is the absence of the Fe XXVI Lyman $\alpha$ lines near $7 \mathrm{keV}$ in the red spectrum, as well as some other lines from the hot plasma. In the black, two-temperature spectrum with Lyman $\alpha$ line is solely produced by the hot gas. In the red, non-thermal spectrum there are simply no Fe XXVI ions so the Lyman $\alpha$ cannot be produced at all.

The most sensitive lines for a $1 \mathrm{keV}$ plasma with a non-thermal tail are the the Fe XXVI Lyman $\alpha$ lines and several of the stronger Fe XVII lines in the $0.7-0.8 \mathrm{keV}$ range. These lines are weaker than for a comparable twotemperature plasma. On the other hand, the Fe XXIV line at $6.662 \mathrm{keV}$ is the best line that becomes stronger.

For a $2 \mathrm{keV}$ plasma, the Fe XXVI Lyman $\alpha$ lines are the best diagnostic with weakening due to the non-thermal electrons, and the higher Rydberg lines of Fe XXV with strengthening of the intensity.

For a $4 \mathrm{keV}$ plasma, however, the Fe XXVI Lyman $\alpha$ lines become stronger when non-thermal electrons are present, compared with a two-temperature plasma. This is mainly due to the fact that the excitation rate for this line is low for $\sim 40 \mathrm{keV}$ electrons. 


\section{Non-equilibrium effects}

Because of an extremely low density $\left(\lesssim 10^{-3} \mathrm{~cm}^{-3}\right)$, the typical mean free path of particles in the ICM is long. In such condition, thermal equilibration between electrons and ions is achieved slowly with the timescale given by

$$
t_{\mathrm{ei}} \sim 7 \times 10^{7}\left(\frac{n_{\mathrm{e}}}{10^{-3} \mathrm{~cm}^{-3}}\right)^{-1}\left(\frac{T_{\mathrm{e}}}{5 \times 10^{7} \mathrm{~K}}\right)^{3 / 2}
$$

(Spitzer 1962). Although this timescale is shorter than the typical cluster age, thermal non-equilibrium could exist in the shock front of merging clusters or cluster outskirt. In addition, the so-called non-equilibrium ionization (NEI) effect, where the ionization degree of ions departs from that in an equilibrium plasma, is also expected in such regions (Akahori \& Yoshikawa, 2010, Wong et al., 2011).

There has been some observational tests for NEI conditions in the ICM. Fujita et al. (2008) analyzed Suzaku data of the Ophiuchus cluster and found that the observed line ratio of $\mathrm{Fe} \operatorname{Ly} \alpha$ to $\mathrm{He} \alpha$ emission is consistent with that predicted by the electron temperature determined from the bremsstrahlung continuum. Similarly, no positive evidence for NEI plasma was found in several merging clusters (e.g., Abell 2146: Russell et al. 2012, CIZA J2242.8+5301). Recently, Suzaku observations of Abell 754 has revealed a signature of the NEI (Inoue et al. , 2016). They found that spectra from the highest-temperature region are well characterized by an NEI plasma with $n_{e} t \sim 4 \times 10^{11} \mathrm{~cm}^{-3} \mathrm{~s}$. From this result, they estimated the elapsed time from the shock heating to be $0.36-76 \mathrm{Myr}$, consistent with the traveling time of a shock to pass through that region.

More recently, high-resolution Hitomi/SXS spectra of the Perseus cluster provided the first opportunity to measure the ion temperature in the ICM using the width of well-resolved emission lines (Hitomi Collaboration et al. 2018). The line-of-sight velocity dispersion due to an isotropic thermal motion of ions is given by $\sigma_{\mathrm{th}}=\sqrt{k T_{\mathrm{ion}} / m_{\mathrm{ion}}}$, which depends on the mass of nuclei. On the other hand, the kinetic broadening due to the turbulence motion $\sigma_{\mathrm{v}}$ is common among the elements. Therefore, we can separate the contributions of $\sigma_{\mathrm{th}}$ and $\sigma_{\mathrm{v}}$ by measuring the widths of different elements. Hitomi Collaboration et al. (2018) used emission lines of $\mathrm{Si}, \mathrm{S}, \mathrm{Ca}$ and Fe, and revealed that the ion temperature is consistent with the electron temperature. Future observations with better sensitivity to lighter elements (e.g., O, Ne) will allow us to investigate the non-equilibrium effects (both thermal and ionization) in a number of clusters.

\section{Charge exchange}

Charge exchange (CX) occurs when neutral and ionized particles mix with each other. One or some electrons can be stripped from the cold atom and bind 

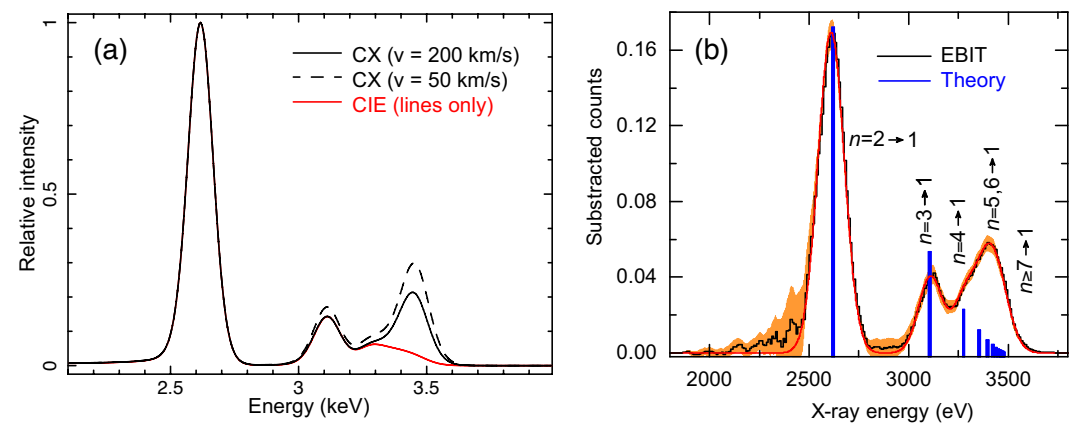

Fig. 7 (a) Model spectrum for charge exchange of bare sulfur with atomic hydrogen, based on the plasma model of Gu et al. (2016), compared with the thermal line emission of hydrogen-like sulfur. (b) Charge exchange emission of bare sulfur with $\mathrm{CS}_{2}$ measured in the electron beam ion trap experiment (Shah et al. 2016). Theoretical predictions of the high-Rydberg-state energies are shown by blue vertical lines.

instead to the hot ion, for the latter often has a deeper ionization potential. The cross section of the process is usually at least two orders of magnitude larger than the free-electron-impact cross section at the $\mathrm{keV}$ energies, making it very effective for ionizing neutral atoms, and for reducing the ionization degree of the hot plasma. In the astrophysical conditions where CX is allowed, it could easily be the dominating radiation process.

The captured electron relaxes to the ground state via line emission. Atomic theory suggests that the electron is likely to drop onto a high Rydberg state with large principle quantum number $n$, creating signature line radiation which appears at a shorter wavelength than those from collisional excitation, but at a longer wavelength than the radiation recombination edge. It also gives a high forbidden-to-resonance ratio for the He $\alpha$ triplet. Recently, accurate and largescale theoretical modeling of CX became available (Stancil, 2001), giving rise to new plasma models in both the AtomDB and SPEX codes (Smith et al., 2012 Gu et al. 2016). Benchmarking these models to the laboratory experiments achieves reasonable agreement (Shah et al., 2016; Cumbee et al., 2018).

Astrophysical observation of the CX phenomena at the X-ray wavelength is traced back to 1996, when comets were discovered as a new class of Xray sources (Lisse et al., 1996). It was soon found that the CX between solar wind and comet atmosphere produces the observed soft X-ray (Cravens, 1997). The presence of solar wind CX from the planet atmosphere (Dennerl et al. 2006; Branduardi-Raymont et al., 2007) and from the heliosphere (Snowden et al. (2004) has also been observationally established. For the extrasolar sources, theoretical studies predicted CX at the cold cloud surface in the hot interstellar medium (Lallement, 2009) and intracluster medium (Lallement, 2004, Gu et al. 2015). Real observations reveals both successes and challenges. There are a growing number of reports of CX-like He $\alpha$ triplet and/or high 1s-np fluxes seen in the X-ray spectra of supernova remnants (e.g., Cygnus loop, Katsuda 
et al. 2011; Cumbee et al. 2014; Roberts \& Wang 2015), star-bursting galaxies (e.g., M 82, Liu et al. 2011, Konami et al. 2011;|Zhang et al. 2014, Cumbee et al. 2016), elliptical galaxies (Pinto et al.| 2016), and clusters of galaxies (Fabian et al. |2011; Walker et al., 2015: Gu et al., 2018). However, the observed line emission is either too dim (Aharonian et al., 2017), or is also explained by other competing processes (Gu et al., 2016).

An intriguing application of CX in clusters of galaxies is to explain the possible line feature at the X-ray energy of $3.5 \mathrm{keV}$. In 2014, two groups claimed to have detected an unknown line feature at around $3.5 \mathrm{keV}$ in the stacked CCD spectra of a large cluster sample (Bulbul et al., 2014, Boyarsky et al., 2014) . The line significance is on the margin, even though the authors managed to enhance the contrast by removing the continuum and known atomic lines from the spectra. The reports attract enormous attention, giving rise to a tide of the publications attempting to explain the possible nature of the unidentified line. The main theoretical focus is towards the possibility that the line is created by the decay of a hypothetical dark matter particle candidate. Recently, $\mathrm{Gu}$ et al. (2015) pointed out an alternative interpretation: the observed line arises from CX between the fully striped sulfur ion and atomic hydrogen, populating a high Rydberg state of the product H-like sulfur ion (Fig. 7). The resulting transitions cannot be found in the present atomic database for thermal plasmas. The two ingredients, the neutral and fully ionized particles, are found to co-exist in galaxy clusters (Conselice et al. 2001). The theoretical calculation of Gu et al. (2015) has been verified with an electron beam ion trap experiment (Shah et al., 2016).

Bulbul et al. (2014) and others reported an anomalously high $3.5 \mathrm{keV}$ line flux in the Perseus cluster compared to the other clusters. The line was expected to be detected with the Hitomi SXS, which is the only instrument capable of fully resolving this feature. By analyzing the Hitomi data of the Perseus center, Aharonian et al. (2017) reported the non-detection of the line at the expected flux, inconsistent with the CCD results at $>99 \%$ confidence level. Instead, the Hitomi spectrum did reveal a hint of the charge exchange feature at $3.44 \mathrm{keV}$ (S XVI) and $8.63 \mathrm{keV}$ (Fe XXV), although the significances are still low (1.6 $\sigma$ for sulfur and $2.4 \sigma$ for iron) with the current data (Hitomi Collaboration et al. 2018). As noted in Aharonian et al. (2017), the central energy of the potential S XVI CX line is about $2.6 \sigma$ away from the best-fit energy of the unknown $3.5 \mathrm{keV}$ line for the XMM-Newton MOS detection (Bulbul et al. 2014). Therefore, it is unclear whether the $3.5 \mathrm{keV}$ line can be fully explained by the sulfur charge exchange, or it still requires other ingredients.

Besides the sulfur and iron, by examining the stacked XMM-Newton RGS data for a sample of 21 cool-core galaxy clusters, Gu et al. (2018) reported the signature of O VIII charge exchange at about $0.84 \mathrm{keV}$ with a significance of $2.8 \sigma$. This result might affect the ICM abundance measurement. If the CX emission is real, the total amount of oxygen in clusters might have been overestimated by up to $20 \%$ through not accounting for charge exchange in previous studies, skewing the star formation model of galaxy clusters. 
A final confirmation of the charge exchange sources in clusters of galaxies has to wait for a more sensitive observation with future spectroscopic instruments. The XRISM mission (launch 2021), equipped with the same microcalorimeter as Hitomi, will provide the first opportunity to detect charge exchange in clusters. Based on the above Hitomi result, the signature Fe XXV high- $n$ transitions might be confirmed at $5 \sigma$ through a 500 ks XRISM observation of the Perseus cluster center, combined with the present Hitomi data of the same object. A same detection confidence will be achieved by a single 100 ks Athena (launch 2030) observation. The region of the charge exchange, as well as some physical parameters, such as the ionization degree and the impinging velocity of hot and cold matter, will be determined with XRISM and Athena.

\section{Progress on atomic codes}

Table 2 Fractional uncertainties on the Hitomi Perseus results

\begin{tabular}{ccccccc}
\hline \hline Effect & Flux & kT & Si abund. & Fe abund. & Turbulence & $N_{\mathrm{H}, \text { hot }}$ \\
\hline statistical $^{\text {a }}$ & 0 & 0 & $5 \%$ & $1 \%$ & $2 \%$ & $7 \%$ \\
plasma code $^{\mathrm{a}}$ & $1 \%$ & $2 \%$ & $19 \%$ & $16 \%$ & $10 \%$ & $40 \%$ \\
multi-temperature $^{\mathrm{b}}$ & $3 \%$ & $4 \%$ & $11 \%$ & $3 \%$ & $1 \%$ & $12 \%$ \\
resonant scattering $^{\mathrm{c}}$ & $1 \%$ & 0 & $5 \%$ & $11 \%$ & $9 \%$ & - \\
NEI $^{\mathrm{c}}$ & $1 \%$ & $1 \%$ & $2 \%$ & 0 & $1 \%$ & $8 \%$ \\
charge exchange $^{\mathrm{c}}$ & 0 & 0 & $2 \%$ & $5 \%$ & $2 \%$ & $7 \%$ \\
effective area cor. $^{\mathrm{d}}$ & $1 \%$ & $2 \%$ & $3 \%$ & $1 \%$ & 0 & $3 \%$ \\
gain cor. & 0 & 0 & $14 \%$ & $10 \%$ & $3 \%$ & $3 \%$ \\
\hline
\end{tabular}

(a) Discrepancies on the fits using SPEX v3.03, APEC v3.0.8, and Chianti v8.0.

(b) Changes on the fits after applying the multi-temperature modeling of the ICM structure.

(c) Changes by including the resonant scattering, non-equilibrium ionization, and charge exchange components.

(d) Changes from the manually corrected effective area (see Hitomi Collaboration et al. (2018) for details).

(e) Changes from the parabolic correction on Hitomi energy scale (see Hitomi Collaboration et al. (2018) for details).

The atomic data and plasma model are essential for analyzing the spectra of astronomical plasma. As mentioned earlier, the study of the detailed spectral features heavily relies on the theoretical calculation of atomic processes. For instance, the excitation and radiative cascade rates are crucial for the resonant scattering measurement ( $\$ 2$, and the sulfur charge exchange line at $\sim 3.5 \mathrm{keV}$ is predicted based on the latest calculation of the electron-capture cross section (\$5). With the advent of high-resolution X-ray spectroscopy instruments, the need for accurate and complete atomic data and plasma model is not just growing but becoming urgent, while the production of the high quality fundamental atomic data is clearly not simple. 

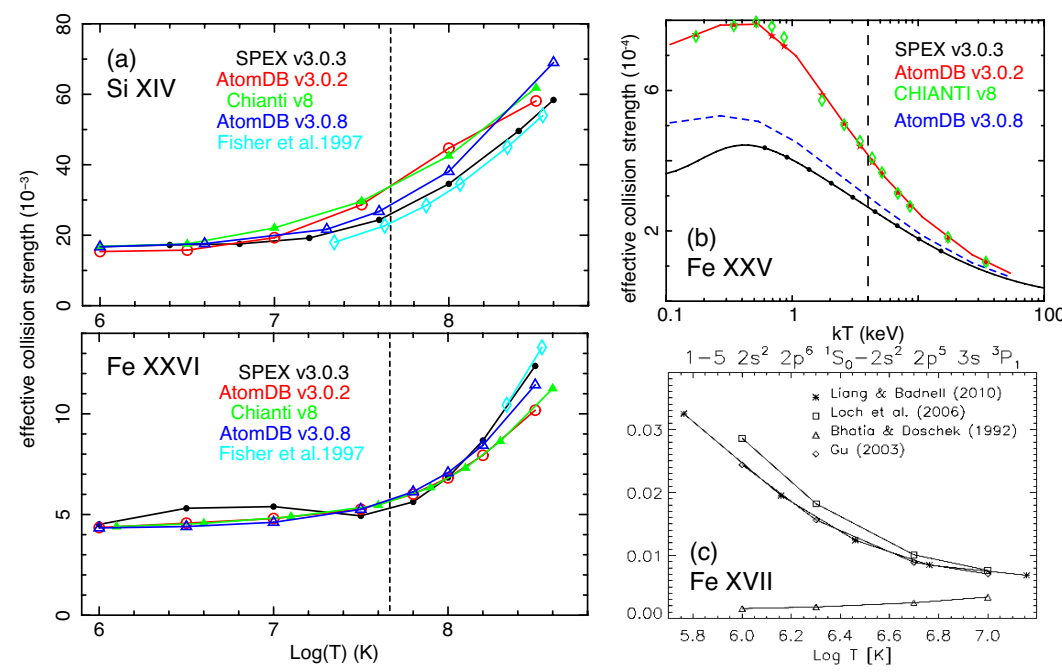

Fig. 8 (a) Comparison of effective collision strength as a function of electron temperature, for combined Ly $\alpha 1$ and Ly $\alpha 2$ transitions. The vertical line shows a temperature of $4 \mathrm{keV}$. (b) Same as (a), but for the $1 \mathrm{~s}^{2}\left({ }^{1} \mathrm{~S}_{0}\right)-1 \mathrm{~s} 2 \mathrm{~s}\left({ }^{3} \mathrm{~S}_{1}\right)$ transition of He-like Fe. (c) Effective collision strength for one of the major Fe XVII lines (Del Zanna 2011).

The Hitomi/SXS spectrum of the Perseus cluster, with $\sim 5 \mathrm{eV}$ resolution in the $2-10 \mathrm{keV}$ band, offers an unprecedented benchmark of the atomic model calculations for a $\sim 4 \mathrm{keV}$ optically-thin thermal plasma. It unveils both successes and challenges of the current plasma codes, the AtomDB/APEC (Smith et al. 2001, Foster et al., 2012) and SPEX (Kaastra et al., 1996). Neither the AtomDB nor SPEX could provide statistically acceptable fits to the Hitomi spectrum with their pre-launch versions (Hitomi Collaboration et al., 2016), immediately revealing inadequacies in atomic data of both codes. A series of updates addressed most of the issues, leading to an overall agreement between the two codes and acceptable fits to the Hitomi data with the latest versions. The remaining issues with the Hitomi data are mostly on the detailed line intensity (Hitomi Collaboration et al. 2018). As shown in Fig. 8, the calculations of the collisional excitation and dielectronic recombination for $\mathrm{H} / \mathrm{He} / \mathrm{Li}$-like ions highly depend on the theoretical models, which do not converge well for a $4 \mathrm{keV}$ plasma. The resulting systematic uncertainties on the Fe abundance is $16 \%$. As shown in Table 2 it is 16 times higher than the statistical uncertainty, and yet significantly higher than the instrumental calibration errors and the errors caused by astrophysical modeling. The final solution to the issue is still waiting for a targeted laboratory benchmark measurement.

The atomic code issues revealed by the Hitomi data are only the tip of the iceberg, as a large portion of the database remains untested. A long-term and continuous effort is required to ensure that plasma codes will be ready to 
face the challenges from the future high-resolution spectroscopy, which will be achieved with the XRISM and the Athena mission. These missions will observe a broad range of cosmic plasmas, including collisionally-ionized plasma with temperatures between $10^{4}-10^{9} \mathrm{~K}$, either optically-thin and optically-thick, and photo-ionized plasma with a similar range of ionization states. The source could be in either ionization equilibrium and non equilibrium. The non-thermal excitation (\$3) and charge exchange (\$5) line emission are also expected from various types of targets.

Among the atomic calculations to be verified, the Fe L-shell spectrum (with Fe XVII to Fe XXIV) is one of the top priorities, as it contains strong emission lines which are crucial for a range of science cases (\$2). To determine where the discrepancies and inadequacies exist, the atomic team need to perform a systematic testing of the present atomic models against each other, as well as against deep observed data in the archives. The recent theoretical calculations with the $R$-matrix method (Liang \& Badnell, 2010, Witthoeft et al., 2007) and improved distorted wave method (Gu, 2003), as well as the experimental data with electron beam ion traps (Bernitt et al. 2012, Hell et al. 2016) will be used for solving these issues. When disagreement remains, new dedicated theoretical calculation and/or targeted laboratory measurements will be required.

\section{Prospects for future missions}

In this paper we have reviewed the X-ray spectroscopic features produced by a range of physical processes in the ICM. Most of them are faint, easily swamped by the thermal radiation from the hot gas, and their presence can only be corroborated by high resolution X-ray spectroscopy. The scientific potential behind these features is enormous, making them valid targets for the future X-ray missions. When the XRISM and Athena will be in orbit, the resonant scattering will be studied for many clusters, and combined with the direct velocity measurement via Doppler broadening, it will provide us a powerful probe of the anisotropy and spatial scales of the ICM random motion. The signature of particle acceleration in merging events will be assessed, for the first time, through measurements of the satellite lines. The timescale needed for the ICM to equilibrate behind a merging and/or accretion shock can be determined by using the NEI modeling. The charge exchange from the cool cores will be measured, providing a new tool to help solve the puzzle of why there are such cold clouds embedded in otherwise hot ICM. The formation of the giant cold gas cloud might be a key to understand the cooling vs heating balance in cluster cores.

The non-dispersive Hitomi Perseus spectrum made us aware of the problems with the present plasma codes in modeling the thermal radiation from the ICM. Before XRISM is ready to launch, these problems will be addressed by a combined effort of atomic code testing, new theoretical calculations, and targeted laboratory measurements. 
Acknowledgements L.G. is supported by the RIKEN Special Postdoctoral Researcher Program. SRON is supported financially by NWO, the Netherlands Organization for Scientific Research.

\section{References}

Aharonian, F. A., Akamatsu, H., Akimoto, F., et al. 2017, The Astrophysical Journal, 837, L15

Ahoranta J., Finoguenov A., Pinto C., Sanders J., Kaastra J., de Plaa J., Fabian A., 2016, Astronomy \& Astrophysics, 592, A145

Akahori, T., \& Yoshikawa, K. 2010, Publications of the Astronomical Society of Japan, 62, 335

Akimoto F., Furuzawa A., Tawara Y., Yamashita K., 1999, Astronomische Nachrichten, 320, 283

Akimoto F., Furuzawa A., Tawara Y., Yamashita K., 2000, Advances in Space Research, 25, 603

Bernitt, S., Brown, G. V., Rudolph, J. K., et al. 2012, Nature, 492, 225

Böhringer H., et al., 2001, Astronomy \& Astrophysics, 365, L181

Boyarsky, A., Ruchayskiy, O., Iakubovskyi, D., \& Franse, J. 2014, Physical Review Letters, 113, 251301

Branduardi-Raymont, G., Bhardwaj, A., Elsner, R. F., et al. 2007, Astronomy \& Astrophysics, 463, 761

Bulbul, E., Markevitch, M., Foster, A., et al. 2014, The Astrophysical Journal, 789,13

Buote D. A., Canizares C. R., Fabian A. C., 1999, Monthly Notices of the Royal Astronomical Society, 310, 483

Chandrasekhar S., 1950, Radiative Transfer, Oxford, Clarendon Press, 1950

Churazov E., Forman W., Jones C., Sunyaev R., Böhringer H., 2004, Monthly Notices of the Royal Astronomical Society, 347, 29

Churazov E., Zhuravleva I., Sazonov S., Sunyaev R., 2010, Space Science Reviews, 157, 193

Conselice, C. J., Gallagher, J. S., III, \& Wyse, R. F. G. 2001, The Astronomical Journal, 122, 2281

Cravens, T. E. 1997, Geophysical Research Letters, 24, 105

Cumbee, R. S., Henley, D. B., Stancil, P. C., et al. 2014, The Astrophysical Journal, 787, L31

Cumbee, R. S., Liu, L., Lyons, D., et al. 2016, Monthly Notices of the Royal Astronomical Society, 458, 3554

Cumbee, R. S., Mullen, P. D., Lyons, D., et al. 2018, The Astrophysical Journal, 852,7

Dennerl, K., Lisse, C. M., Bhardwaj, A., et al. 2006, Astronomy \& Astrophysics, 451, 709

Dupke R. A., Arnaud K. A., 2001, The Astrophysical Journal, 548, 141

Ezawa H., et al., 2001, Publications of the Astronomical Society of Japan, 53, 595 
Fabian, A. C., Sanders, J. S., Williams, R. J. R., et al. 2011, Monthly Notices of the Royal Astronomical Society, 417, 172

Foster, A. R., Ji, L., Smith, R. K., \& Brickhouse, N. S. 2012, The Astrophysical Journal, 756, 128

Fujita, Y., Hayashida, K., Nagai, M., et al. 2008, Publications of the Astronomical Society of Japan, 60, 1133

Gabriel, A. H. \& Phillips, K. J. H. 1979, Monthly Notices of the Royal Astronomical Society, 189, 319

Gastaldello F., Molendi S., 2004, The Astrophysical Journal, 600, 670

Gilfanov M. R., Sunyaev R. A., Churazov E. M., 1987, Soviet Astronomy Letters, 13,3

Gu, M. F. 2003, The Astrophysical Journal, 582, 1241

Gu, M. F., Beiersdorfer, P., Brown, G. V., et al. 2012, Canadian Journal of Physics, 90, 351

Gu, L., Kaastra, J., Raassen, A. J. J., et al. 2015, Astronomy \& Astrophysics, 584, L11

Gu, L., Kaastra, J., \& Raassen, A. J. J. 2016, Astronomy \& Astrophysics, 588, A52

Gu, L., Mao, J., Costantini, E., \& Kaastra, J. 2016, Astronomy \& Astrophysics, 594, A78

Gu, L., Mao, J., de Plaa, J., et al. 2018, Astronomy \& Astrophysics, 611, A26

Hahn, M., \& Savin, D. W. 2015, The Astrophysical Journal, 809, 178

Hamilton D. R., 1947, The Astrophysical Journal, 106, 457

Hell, N., Brown, G. V., Wilms, J., et al. 2016, IAU Focus Meeting, 29, 295

Hitomi Collaboration, Aharonian, F., Akamatsu, H., et al. 2016, Nature, 535, 117

Hitomi Collaboration, Aharonian, F., Akamatsu, H., et al. 2017, Nature, 551, 478

Hitomi Collaboration, Aharonian, F., Akamatsu, H., et al. 2018, Publications of the Astronomical Society of Japan, 70, 10

Hitomi Collaboration, Aharonian, F., Akamatsu, H., et al. 2018, Publications of the Astronomical Society of Japan, 70, 11

Hitomi Collaboration, Aharonian, F., Akamatsu, H., et al. 2018, Publications of the Astronomical Society of Japan, 70, 12

Inoue, S., Hayashida, K., Ueda, S., et al. 2016, Publications of the Astronomical Society of Japan, $68, \mathrm{~S} 23$

Kaastra, J. S., Mewe, R., \& Nieuwenhuijzen, H. 1996, UV and X-ray Spectroscopy of Astrophysical and Laboratory Plasmas, 411

Kaastra J. S., Lieu R., Mittaz J. P. D., Bleeker J. A. M., Mewe R., Colafrancesco S., Lockman F. J., 1999, The Astrophysical Journal, 519, L119

Kaastra J. S., Bykov, A. M. \& Werner, N., 2009, Astronomy \& Astrophysics, 503,373

Kahn S. M., et al., 2003, Matter and Energy in Clusters of Galaxies, ASP Conference Proceedings, 301, 23

Katsuda, S., Tsunemi, H., Mori, K., et al. 2011, The Astrophysical Journal, 730,24 
Konami, S., Matsushita, K., Tsuru, T. G., Gandhi, P., \& Tamagawa, T. 2011, Publications of the Astronomical Society of Japan, 63, S913

Lallement, R. 2004, Astronomy \& Astrophysics, 422, 391

Lallement, R. 2009, Space Science Reviews, 143, 427

Liang, G. Y., \& Badnell, N. R. 2010, Astronomy and Astrophysics, 518, A64

Lisse, C. M., Dennerl, K., Englhauser, J., et al. 1996, Science, 274, 205

Liu, J., Mao, S., \& Wang, Q. D. 2011, Monthly Notices of the Royal Astronomical Society, 415, L64

Mathews W. G., Buote D. A., Brighenti F., 2001, The Astrophysical Journal, 550, L31

Mitchell, R. J., Culhane, J. L., Davison, P. J. N., \& Ives, J. C. 1976, Monthly Notices of the Royal Astronomical Society, 175, 29P

Molendi S., Matt G., Antonelli L. A., Fiore F., Fusco-Femiano R., Kaastra J., Maccarone C., Perola C., 1998, The Astrophysical Journal, 499, 608

Mushotzky, R., Loewenstein, M., Arnaud, K. A., et al. 1996, The Astrophysical Journal, 466, 686

Ogorzalek A., et al., 2017, Monthly Notices of the Royal Astronomical Society, 472, 1659

Panagoulia E. K., Sanders J. S., Fabian A. C., 2015, Monthly Notices of the Royal Astronomical Society, 447, 417

Peterson, J. R., Paerels, F. B. S., Kaastra, J. S., et al. 2001, Astronomy and Astrophysics, 365, L104

Pinto, C., Fabian, A. C., Ogorzalek, A., et al. 2016, Monthly Notices of the Royal Astronomical Society, 461, 2077

de Plaa J., Zhuravleva I., Werner N., Kaastra J. S., Churazov E., Smith R. K., Raassen A. J. J., Grange Y. G., 2012, Astronomy and Astrophysics, 539, A34

Rephaeli, Y., Nevalainen, J., Ohashi, T. \& Bykov, A. M., 2008, Space Science Reviews, 134, 71

Roberts, S. R., \& Wang, Q. D. 2015, Monthly Notices of the Royal Astronomical Society, 449, 1340

Russell, H. R., McNamara, B. R., Sanders, J. S., et al. 2012, Monthly Notices of the Royal Astronomical Society, 423, 236

Sakelliou I., et al., 2002, Astronomy and Astrophysics, 391, 903

Sanders J. S., Fabian A. C., 2006, Monthly Notices of the Royal Astronomical Society, 370, 63

Sazonov S. Y., Churazov E. M., Sunyaev R. A., 2002, Monthly Notices of the Royal Astronomical Society, 333, 191

Shah, C., Dobrodey, S., Bernitt, S., et al. 2016, The Astrophysical Journal, 833,52

Shang C., Oh S. P., 2013, Monthly Notices of the Royal Astronomical Society, 433, 1172

Smith, R. K., Brickhouse, N. S., Liedahl, D. A., \& Raymond, J. C. 2001, The Astrophysical Journal Letters, 556, L91

Smith, R. K., Foster, A. R., \& Brickhouse, N. S. 2012, Astronomische Nachrichten, 333, 301 
Snowden, S. L., Collier, M. R., \& Kuntz, K. D. 2004, The Astrophysical Journal, 610,1182

Stancil, P. C. 2001, Spectroscopic Challenges of Photoionized Plasmas, 247, 3

Takahashi, T., Kokubun, M., Mitsuda, K., et al. 2016, Proceedings of the SPIE, 9905, 99050U

Walker, S. A., Kosec, P., Fabian, A. C., \& Sanders, J. S. 2015, Monthly Notices of the Royal Astronomical Society, 453, 2480

Werner N., Zhuravleva I., Churazov E., Simionescu A., Allen S. W., Forman W., Jones C., Kaastra J. S., 2009, Monthly Notices of the Royal Astronomical Society, 398, 23

Witthoeft, M. C., Whiteford, A. D., \& Badnell, N. R. 2007, Journal of Physics B Atomic Molecular Physics, 40, 2969

Wong, K.-W., Sarazin, C. L., \& Ji, L. 2011, The Astrophysical Journal, 727, 126

Xu H., et al., 2002, The Astrophysical Journal, 579, 600

Del Zanna, G. 2011, Astronomy and Astrophysics, 536, A59

Zhang, S., Wang, Q. D., Ji, L., et al. 2014, The Astrophysical Journal, 794, 61

Zhuravleva I. V., Churazov E. M., Sazonov S. Y., Sunyaev R. A., Forman W., Dolag K., 2010, Monthly Notices of the Royal Astronomical Society, 403, 129

Zhuravleva I. V., Churazov E. M., Sazonov S. Y., Sunyaev R. A., Dolag K., 2011, Astronomy Letters, 37, 141

Zhuravleva I., et al., 2013, Monthly Notices of the Royal Astronomical Society, 435,3111 